\title{
Trombosis de la vena umbilical como complicación de pancreatitis aguda. Reporte de un caso
}

\section{Thrombosis of umbilical vein, as a complication of acute pancreatitis.}

\section{Case report}

Edgar Ferrer-Alpuin* y Omar Salmerón-Covarrubias

Servicio de Radiología, Hospital General Dr. Miguel Silva, Morelia, Michoacán, México

\section{RESUMEN}

La patología de la vena umbilical en adultos es sumamente rara y los casos reportados de trombosis en ella se asocian a trombosis de la vena porta, la cual a su vez es una complicación secundaria a pancreatitis aguda. A continuación, se presenta el caso clínico de una paciente que acudió a nuestro hospital por un cuadro de pancreatitis aguda de aparente origen biliar. Al realizarle una tomografía abdominal para la estadificación de la pancreatitis, se observó una trombosis de la vena umbilical y de la vena porta como complicaciones secundarias.

Palabras clave: Vena umbilical. Trombosis. Pancreatitis. Vena porta.

\section{ABSTRACT}

Umbilical vein pathology is extremely rare and reported cases of thrombosis at this level are associated with portal vein thrombosis, which in turn is a secondary complication of acute pancreatitis. Below is a clinical case of a patient who came to our hospital for acute pancreatitis of apparent biliary origin and who performed an abdominal tomography for staging pancreatitis, a thrombosis of the umbilical vein and portal vein is observed as secondary complications.

Key words: Umbilical vein. Thrombosis. Pancreatitis. Portal vein. 


\section{INTRODUCCIÓN}

Durante la etapa fetal, la vena umbilical es la encargada de transportar la mayoría de la sangre oxigenada hacia el conducto venoso para posteriormente drenar hacia la vena cava inferior ${ }^{1}$. Una vez que las funciones pulmonares, digestivas y renales se desarrollan, la vena umbilical se colapsa y se aloja en el ligamento falciforme. Este ligamento tiene como función principal unir el hígado a la pared anterior del cuerpo y separar el lóbulo izquierdo en sus segmentos lateral y medial $^{2}$. Los casos reportados de trombosis de la vena umbilical se asocian a trombosis de la vena porta. Las enfermedades malignas, la pancreatitis y el trasplante hepático son factores de riesgo para trombosis de las venas umbilical y portal ${ }^{3}$. Es importante recordar que los cálculos biliares son la principal causa de pancreatitis aguda en los países desarrollados, representando hasta un $75 \%$ de los casos, y un $10 \%$ de estos pacientes desarrollarán complicaciones extrapancreáticas, siendo las trombosis de la vena porta y de la vena umbilical causas poco frecuentes ${ }^{4}$.

Los estudios de imagen desempeñan un papel importante en esta patología. La ecografía permite valorar la ausencia de flujo con la técnica de Doppler color, mientras que la tomografía con contraste intravenoso es el método de imagen elegido para identificar de manera general todas las complicaciones asociadas a pancreatitis aguda ${ }^{2-5}$.

Los pacientes que presenten una trombosis portal, ya sea asociada a trombosis de la vena umbilical o no, deben recibir tratamiento anticoagulante oral o intravenoso posterior a cualquier procedimiento quirúrgico, siempre y cuando no presenten cirrosis ${ }^{6}$.

\section{PRESENTACIÓN DEL CASO}

Mujer de 57 años que acude a nuestro hospital por dolor abdominal de tipo opresivo en el epigastrio, referido con una intensidad de 9/10 en la escala del dolor, el cual no mejora con la ingesta de analgésicos (no especificado), acompañado de náuseas y vómitos de contenido alimenticio de 2 días de evolución. A su ingreso, el servicio de urgencias solicita laboratorios que muestran unos valores de amilasa de 471 U/1 y de lipasa de 345 U/1. Debido a esto, y por protocolo, se solicitan ultrasonido abdominal y tomografía de abdomen en fase simple y contrastada para abordaje de pancreatitis aguda, observando una lesión hipodensa a nivel de la vena umbilical, así como dilatación de esta (Fig. 1); en la fase contrastada se identifica una ausencia de realce de dicha lesión con el medio de contraste (Fig. 2). Al identificar estos hallazgos se decidió realizar ultrasonido Doppler dúplex, que mostró la ausencia de flujo en la vena umbilical y en la vena porta (Fig. 3). Se solicitó resonancia magnética, en la que se apreció la persistencia de dicha lesión, la cual se comportaba hipointensa en T1 y T2 (Figs. 4 y 5). Posterior a los estudios de imagen, se realizó una evaluación prequirúrgica para planificar una colecistectomía abierta por parte del servicio de cirugía general, la cual se realizó sin complicaciones. Después del procedimiento quirúrgico se indicó ayuno y control de enzimas pancreáticas hasta su egreso hospitalario, y se agregó anticoagulación oral (warfarina, $2 \mathrm{mg}$ /día por 5 días). La paciente cursó asintomática durante la etapa posquirúrgica. 


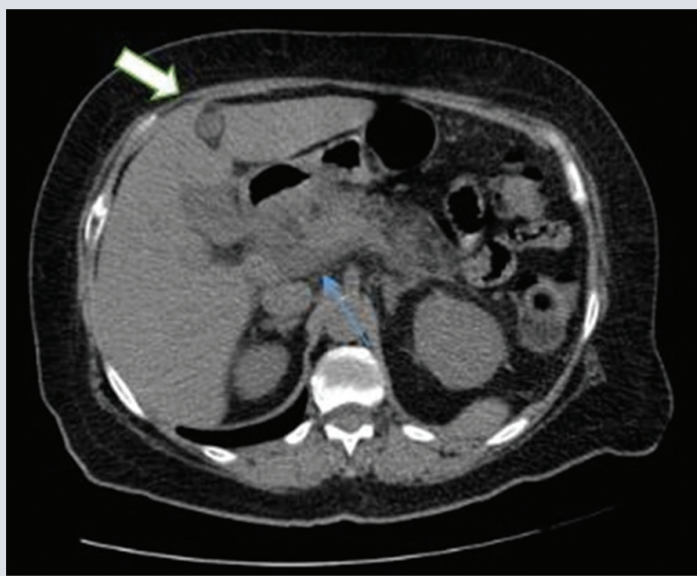

Figura 1. Tomografía de abdomen simple en corte axial en la cual se observa una imagen ovoide hipodensa al parénquima hepático a nivel de la vena umbilical (flecha blanca). Se aprecia líquido adyacente a nivel de la cabeza del páncreas (flecha azul).

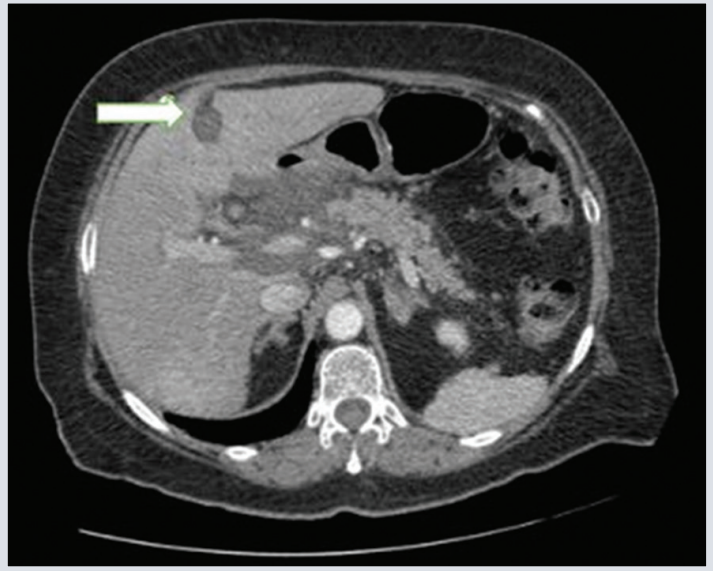

Figura 2. Tomografía de abdomen en fase contrastada en la que se observa la ausencia de realce a nivel de la vena umbilical (flecha).

\section{CONCLUSIONES}

La pancreatitis aguda se presenta en una tasa relativamente alta en nuestro país, con cifras de 5-11 casos por cada 100,000
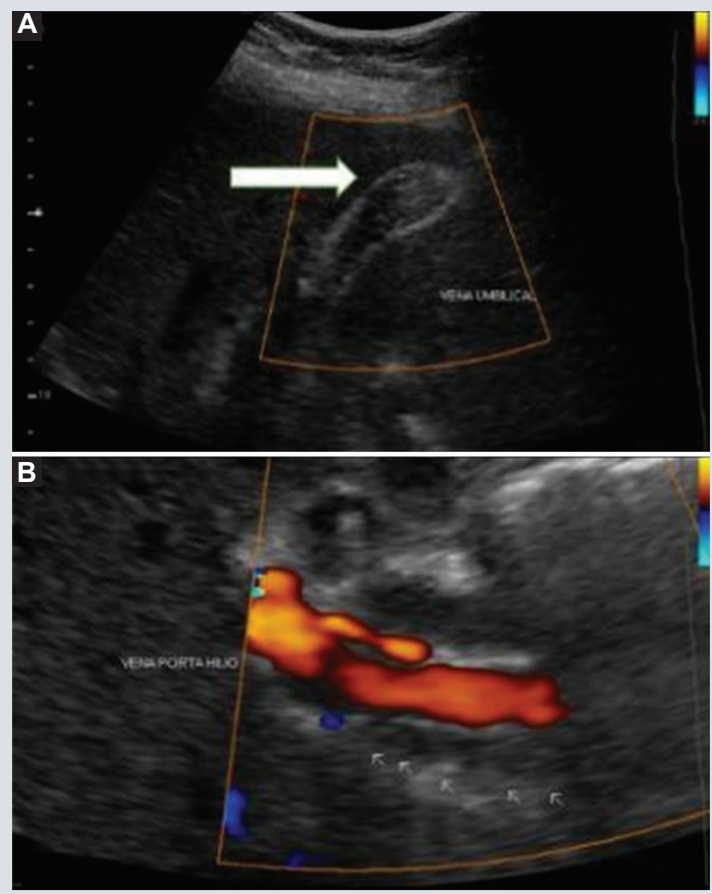

Figura 3. A: ultrasonido Doppler de la vena umbilical en el que se observa la ausencia de flujo a la aplicación de la señal Doppler color (flecha). De igual manera se identifica la dilatación de la vena umbilical. B: ultrasonido Doppler de la vena porta en el que se observa un defecto de llenado parcial en relación con un trombo (flechas).

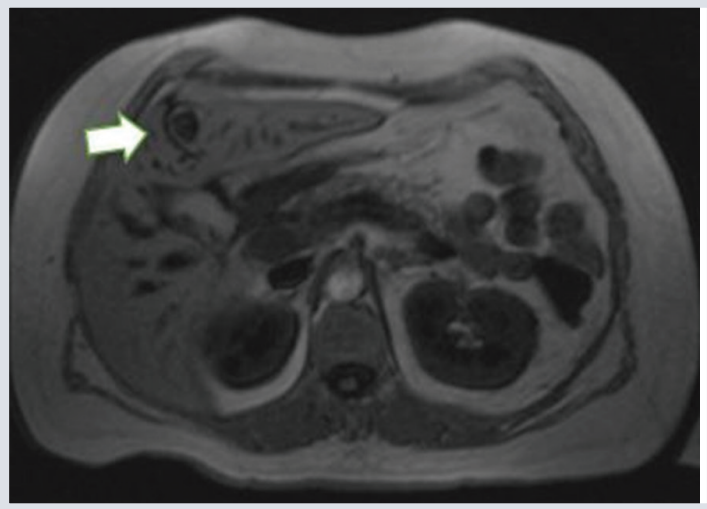

Figura 4. Colangiorresonancia ponderada en T1, corte axial, en la que se observa una imagen hipointensa a nivel de la vena umbilical (flecha).

habitantes, y hasta un $10 \%$ de estos pacientes sufren complicaciones. La trombosis de 


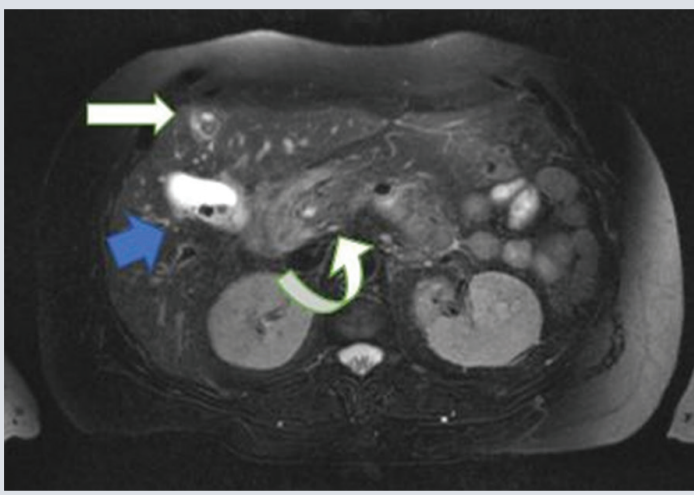

Figura 5. Colangiorresonancia ponderada en T2, corte axial, en la que se observa una imagen hipointensa a nivel de la vena umbilical (flecha blanca). De la misma forma, se observan imágenes con ausencia de señal dentro de la vesícula biliar relacionadas con litos (flecha azul). Se observa líquido peripancreático a nivel de la cabeza del páncreas (flecha curva). el esquema terapéutico conjunto hacia un buen pronóstico.

\section{CONFLICTO DE INTERESES}

Los autores declaran no tener conflictos de intereses.

\section{FINANCIAMIENTO}

El presente caso clínico no presento financiamiento por parte de alguna institución pública o privada para su realización.

\section{RESPONSABILIDADES ÉTICAS}

la vena umbilical, que es una afección sumamente rara, así como la trombosis de la vena porta, son algunas de las complicaciones que pueden asociarse a los eventos de pancreatitis aguda. Su diagnóstico oportuno, previo a un evento terapéutico, facilita la planeación de este y evita las consecuencias que estas complicaciones pueden llegar a tener. Por ello, es importante compartir los hallazgos de este caso para que se conozca una de las diferentes formas de presentación de una complicación del proceso inflamatorio de la glándula pancreática. De igual manera, hay que hacer énfasis en que, debido a que la trombosis de la vena umbilical se produce en la mayoría de los casos como una complicación de otra enfermedad de base, es necesario estabilizar primero el cuadro principal del paciente y luego agregar anticoagulantes orales o por vía intravenosa durante la estancia hospitalaria, con el fin de disminuir el cuadro trombótico y guiar
Protección de personas y animales. Los autores declaran que los procedimientos seguicomité de experimentación humana responsable y de acuerdo con la Asociación Médica Mundial y la Declaración de Helsinki.

Confidencialidad de los datos. Los autores declaran que han seguido los protocolos de su centro de trabajo sobre la publicación de datos de pacientes.

Derecho a la privacidad y consentimiento informado. Los autores han obtenido el consentimiento informado de los pacientes y/o sujetos referidos en el artículo. Este documento obra en poder del autor de correspondencia.

\section{BIBLIOGRAFÍA}

1. Lim HQC, Lee XWJ, Mathias N. A rare finding of falciform ligament thrombosis as a sequel of acute pancreatitis. Case Rep Radiol. 2017;2017:2879568. dos se conformaron a las normas éticas del 
2. Martínez Chamorro E, Ibáñez Sanz L, Depetris M, Encinas Escobar P, Borruel Nacenta S. Focal fat infarction of the falciform ligament. Eurorad. 2018; Case: 15983.

3. Seeger M, Gunter R, Hinrichsen H, Both M, Helwig U, Artt A, et al. Chronic portal vein thrombosis: transcapsular hepatic colateral vessels and communicating ectopic varices. Radiology. 2010;257:568-78.

4. Garber A, Frakes C, Arora Z, Chahal P. Mechanisms and management of acute pancreatitis. Gastroenterol Res Pract. 2018;2018:6218798.
5. Ahmad RS, Jabeen H, Adnan M. Complications of early cholecystectomy in acute biliary pancreatitis. JMMC. 2018;9(1):42-44. Disponible en: http://www.jmmc.mmc.edu.pk/index.php/JMMC/article/download $/ 53 / 43$.

6. Boyer TD, Schiff ER. Management of portal vein thrombosis. Current developments in the treatment of hepatitis and hepatobiliary disease. Gastroenterol Hepatol. 2008;4(10):699-700. 